\title{
Network Lifetime Analysis of Routing Protocols of Short Network in Qualnet
}

\author{
Pratyush Pandey, Er.Ashish Das, Prof.A.K.Jaiswal \\ M.tech, ECE, Dept. of Electronic Science, HOD. Dept. of Electronic, SHIATS Deemed to be University \\ ALLAHABAD-07, U.P, INDIA
}

\begin{abstract}
A Mobile Ad-Hoc Network (MANET) is a collection of wireless mobile nodes that communicates with each other without using any existing infrastructure, access point or centralized administration. Mobile ad-hoc network have the attributes such as wireless connection, continuously changing topology, distributed operation and ease of deployment. In this paper we have compared the energy consumption of reactive, proactive \& hybrid routing protocol AODV,DSR,RIP \& ZRP by using different mobility model. We have analyzed the Network lifetime of protocols by varying pay load, mobility, pause time and type of traffic (CBR). A detailed simulation has been carried out in qualnet. The metrics used for performance analysis are energy consumed \& battery consumption. It has been observed that RIP has better network lifetime than other.
\end{abstract}

\section{Introduction}

A Wireless ad hoc network is a collection of self organized wireless mobile nodes dynamically forming a temporary network without the aid of any established or fixed infrastructure and centralized administration control stations, unlike cellular wireless networks. The surrounding physical environment significantly attenuates and distorts the radio transmissions since signal quality degrades with distance. Wireless networks in all their different forms such as mobile ad hoc network (MANET) and vehicular ad hoc network (VANET), wireless sensor network(WSN), wireless mesh network(WMN), etc are coming under this category. In multihop ad hoc network destination nodes may be multiple hops away from the source node. This approach provides a number of advantages as compare to single-hop networking solution. Some of its advantages are (i) support for self configuration and adaption at low cost, (iii) support of load balancing for increasing network life, (iv) greater network flexibility, connectivity, However irrespective of these advantages it also suffered with many challenges associated with restricted battery capacity, unpredictable mobility, routing, etc. [7] ,[5],[6]

MANETs differ from wired Internet Protocol (IP) networks in several respects. Ad hoc networks lack the centralized infrastructure found in both cellular and fixed networks. Nodes and infrastructure may be highly mobile. Second, there is a blurring of IP's typical distinction between routers and hosts. Third, most military MANETs have low bandwidth (kbps) wireless links and batteryoperated nodes that require power-efficient operation.

With a proper analysis of battery consumptions, light weight applications, efficient network protocol and interface power consumption of wireless network can be properly addressed. Flooding based routing protocols rely on message forwarding by broadcasting the message. This mechanism consumes a major portion of battery power at node level also affect the longevity of the network. Energy efficient routing protocols apply some techniques to reduce flooding mechanism by some probabilistic and heuristic based approach but are suffered with increase end-to-end delay and decrease network throughput. For this reason there must be some threshold between power consumptions and other network parameters while designing routing protocols for MANET. In the literature different techniques are proposed to find the energy efficiency of routing protocol, but network lifetime is not properly addressed at different network traffic, load and mobility. Focusing on these three parameters we made an attempt to determine the network lifetime of AODV, DSR, RIP and ZRP at mobility, pause time and load. AODV and DSR represent the reactive category of routing mechanism,RIP represent proactive and ZRP represent the hybrid approach of routings routing in ad hoc network. [10]

The rest of the paper is organised as follows. In Section 2, we present routing protocols of MANETs under which we discuss about AODV, DSR,RIP and ZRP. In Section 3, we discuss the network lifetime parameter and simulation environments. In Section 4 simulation results are discussed at different network conditions. In Section 5, we end our discussion with conclusion and thought for future work on this topic. 


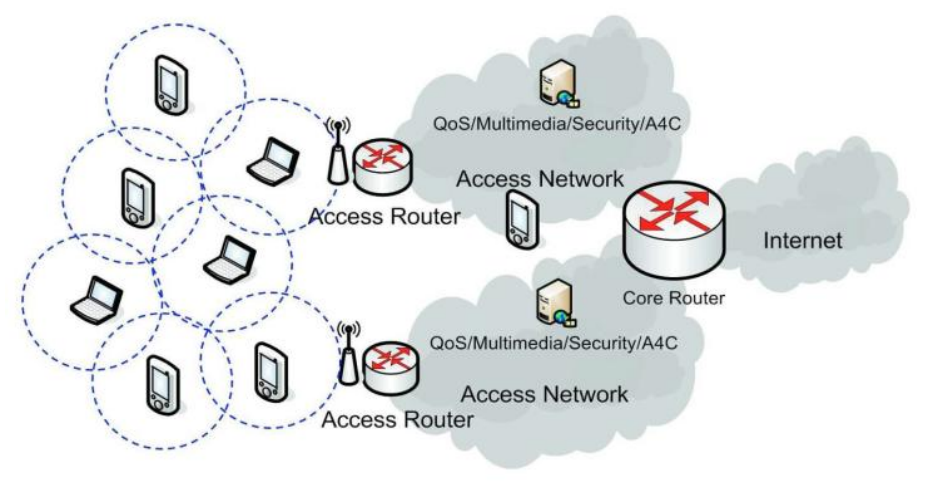

Ad-hoc network architecture

\section{Routing Protocols}

.Routing protocols are divided into three categories namely,proactive and reactive $\&$ hybrid

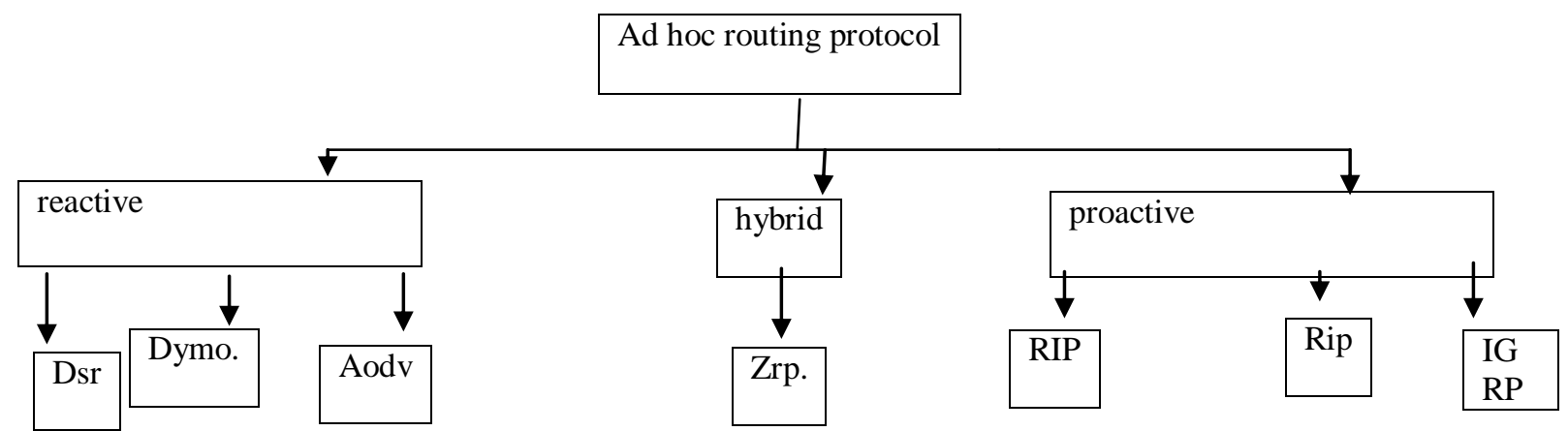

In Reactive routing protocol routing information is acquired on-demand. Reactive routing protocols use two different operations to Route discovery and Route maintenance operation. Route maintenance is the process of responding to change in topology that happen after a route has initially been created, Route Maintenance is used to handle route breaks [8]. Examples: AODV, DSR, DYMO, LAR1

Proactive routing protocols maintain information continuously. Typically, a node has a table containing information on how to reach every other node and the algorithm tries to keep this table up-to-date. Change in network topology is propagated throughput the network.

Hybrid routing protocols are a new generation of protocol, which are both are Proactive and Reactive in nature. Most hybrid protocols proposed to date are zone based, which means that the network is partitioned or seen as a number of zones by each node. Normally, Hybrid routing protocols for MANETs exploit hierarchical network architectures. [4]

\section{AODV}

AODV protocol is specially used for mobile ad hoc networks. It provides a quick adaptation to dynamic link condition, link fault, low processing and memory usage overhead. It enables dynamic, self-ripting, multihop routing between participating mobile nodes wishing to establish and maintain an ad hoc network.AODV allows mobile nodes to obtain routes quickly for new destinations, and does not require nodes to maintain routes to destinations that are not in active communication. AODV[9] allows mobile nodes to respond to link breakages and changes in network topology in a timely manner. It uses sequence numbers to prevent routing loops.

\section{Dynamic Source Routing (DSR)}

Dynamic Source Routing (DSR) [3] is a routing technique in which the sender of a packet determines the complete sequence of nodes through which the packet has to pass; the sender unambiguously lists this route in the packets header, identifying each forwarding "hop" by the address of the next node to which to transmit the packet on its way to the destination host. It also computes the routes when necessary and then maintains them. The protocol is composed of the two main mechanisms of "Route Discovery" and "Route Maintenance", which work together to allow nodes to discover and maintain routes to arbitrary destinations in the ad hoc network. All aspects of the protocol operate entirely on demand, allowing the routing packet overhead of DSR 
to scale automatically to only what is needed to react to changes in the routes currently in use.[The Dynamic Source Routing Protocol for Mobile Ad Hoc Networks (DSR).

\section{Routing Information Protocol (RIP)}

The Routing Information Protocol (RIP) is a distance-vector routing protocol, which employs the hop count as a routing metric. RIP prevents routing loops by implementing a limit on the number of hops allowed in a path from the source to a destination. The maximum number of hops allowed for RIP is 15 . This hop limit, however, also limits the size of networks that RIP can support. A hop count of 16 is considered an infinite distance and used to deprecate inaccessible, inoperable, or otherwise undesirable routes in the selection process. RIP[1] implements the split horizon, route poisoning and hold-down mechanisms to prevent incorrect routing information from being propagated.

\section{Zone Routing Protocol (ZRP):}

The hybrid approach combines the table-driven and source-initiated on-demand driven approaches such that the overhead incurred in route discovery and maintenance is minimized while the efficiency is maximized. The Zone Routing Protocol (ZRP) partitions the network implicitly into zones, where a zone of a node includes all nearby nodes within the zone radius defined in hops. It applies proactive strategy inside the zone and reactive strategy outside the local zone. Each node may potentially be located in many zones. ZRP consists of two subprotocols. The proactive intra zone routing protocol (IARP) is an adapted distance-vector algorithm. When a source has no IARP route to a destination, it invokes a reactive inter-zone routing protocol (IERP), which is very similar to DSR. [2]

\section{Simulation Platform And Models}

In this work Qualnet 6.1 network simulator has been used to evaluate the performance of proactive (RIP), reactive (AODV, DSR) and hybrid (ZRP) routing protocols of mobile ad-hoc networks. The physical medium used is 802.11 PHY with a data rate of 2 Mbps. The MAC protocol used is the 802.11 MAC protocol, configured for MANET mode. In this work wireless module of IEEE $802.11 \mathrm{~b}$ is used to enable mobility of the wireless nodes. IEEE $802.11 \mathrm{~b}$ support more accurate wireless models for propagation, path loss, multipath fading and reception on wireless networks. The simulations are carried out for network densities of 50 nodes respectively. The area considered is $1500 \mathrm{~m} \mathrm{X} 1500 \mathrm{~m}$ for stationary nodes and nodes with mobility of $10 \mathrm{mps}$. Simulations are configured for the performance evaluation of different routing protocols with the metrics like battery capacity \& energy consumed at the destination for stationary and nodes with mobility of $10 \mathrm{mps}$ respectively. Comparison of routing protocols constant bit rate (CBR) traffic patterns are used. The network contains variable CBR traffic connections and packet size of 512 bytes. Packets are send from source nodes in the $0.25 \mathrm{~s}$ interval.

Table 1. Scenario Parameters

\begin{tabular}{|c|c|c|c|}
\hline Routing protocols & \multicolumn{3}{|c|}{ AODV,DSR, RIP \& ZRP } \\
\hline Radio type & \multicolumn{3}{|c|}{$802.11 \mathrm{~b}$} \\
\hline Coordinate System & \multicolumn{3}{|c|}{ Cartesian } \\
\hline Channel frequency & \multicolumn{3}{|c|}{$2.4 \mathrm{GHz}$} \\
\hline Mobility & Group & Random Way Point & None \\
\hline Mobility speeds & \multicolumn{2}{|l|}{ None } & 0 to $10 \mathrm{mps}$ \\
\hline Path loss model & \multicolumn{3}{|c|}{ Two Ray } \\
\hline Energy model & \multicolumn{3}{|c|}{ generic } \\
\hline Shadowing model & \multicolumn{3}{|c|}{ Constant } \\
\hline Pause time & $30 \mathrm{~s}$ & $20 \mathrm{~s}$ & $10 \mathrm{~s}$ \\
\hline Simulation time & \multicolumn{3}{|c|}{300 second } \\
\hline Battery model & \multicolumn{3}{|c|}{ Linear model } \\
\hline Simulation area & \multicolumn{3}{|c|}{$1500 \mathrm{~m} \times 1500 \mathrm{~m}$} \\
\hline Number of nodes & \multicolumn{3}{|l|}{50} \\
\hline Simulation time & \multicolumn{3}{|c|}{$300 \mathrm{~s}$} \\
\hline Simulator & \multicolumn{3}{|c|}{ Qualnet 6.1 } \\
\hline
\end{tabular}




\section{SIMULATIONS - SCENARIOS}

\section{Network Lifetime at Varying Mobility Model}

We consider Random way point mobility (RWP),Group mobility and None (no mobility)We simulate the network at 50nodes. In order to find the best mobility model we fix the CBR connection and pause time of each node. Extensive simulations are done to find the network lifetime of AODV, DSR,RIP and ZRP at three mobility model.. In order to find the best mobility model we fix the CBR connection and pause time of each node. We found that irrespective of type of mobility pattern RIP gives better network life in all scenarios. Figure shows the lifetime analysis of AODV, DSR,RIP and ZRP at node 50. In the rest of our analysis we only consider RWP mobility. For our convienience we change the axis from 0-1200 to 1197.9- 1198.5 because we take the simulation time of 300s.

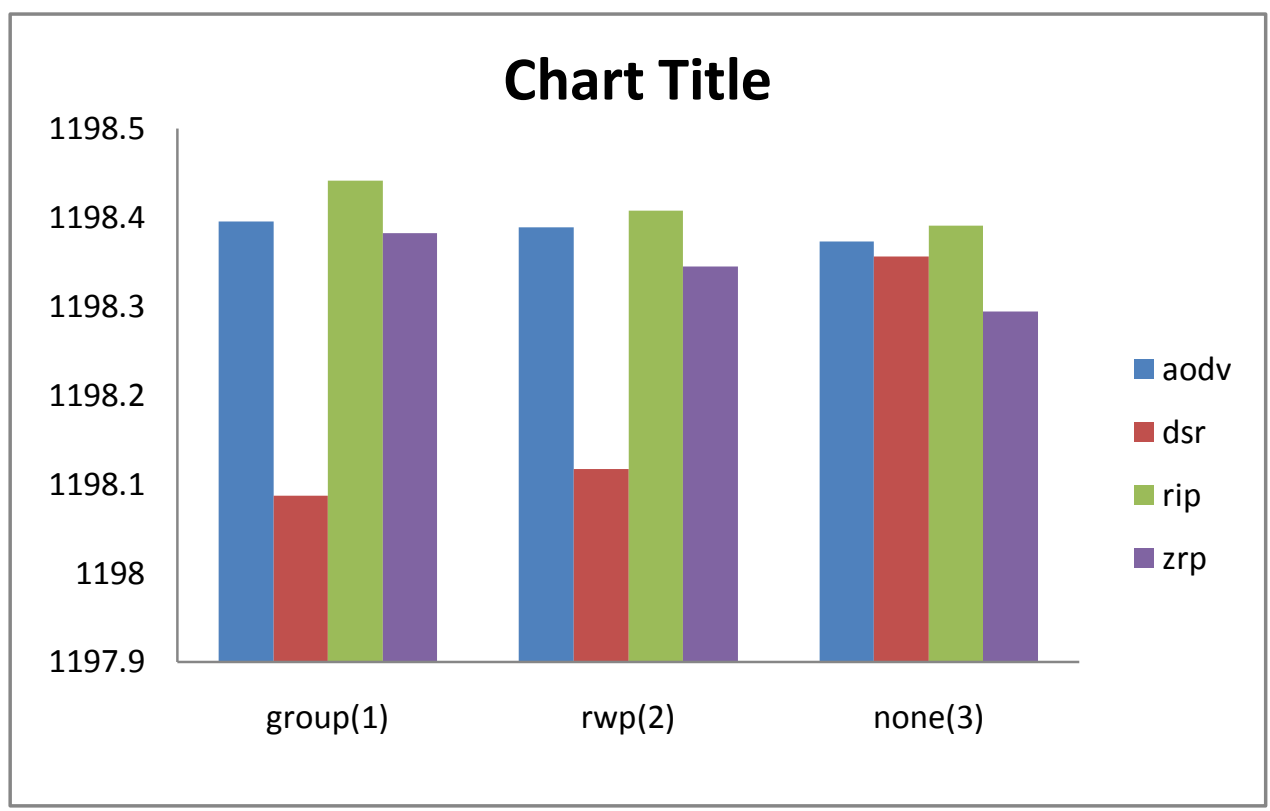

\section{Network Lifetime at Varying CBR Connections}

The Figure shows network lifetime at 50 nodes with varying CBR connections.We consider RWP model with $7 \& 10$ CBR connection. We study the behaviour between network traffic and load. In all case RIP is performs better. The life time of ZRP is always low as compare to DSR, AODV\& RIP. The improve performance of RIP is its greater compatibility with mobility short network size. For our convienience we change the axis from 0-1200 to 1197.9- 1198.5 because we take the simulation time of 300s.

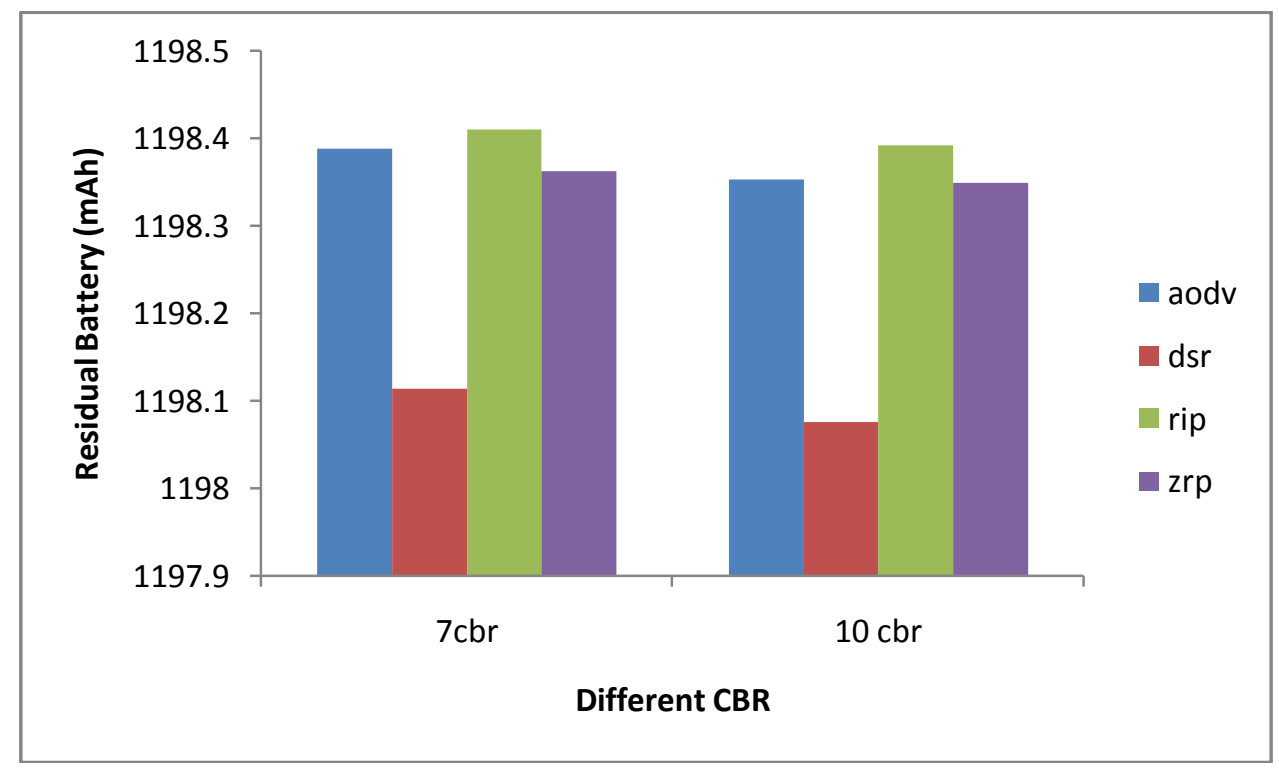




\section{Network Lifetime at Varying Pause Time}

We have already discusses the simulation results of mobility models. For pause time variations we consider RWP for our comparisons. In RWP model node remains stationary for a certain periods of time (known as pause time). The node moves to the destination at a speed in the range [0, max]. When node reaches the destination it waits for time equal to pause time and ripts moving for another destination. It repeats this performance for the entire simulation time. We simulate with three different pause times: 10s, 20s, and 30s. Fig shows the network life time at various pause times at different node density. In all results it is also found that RIP performing better as compared to AODV, DSR and ZRP. It is observed that pause time of the node doesn't make more impact on the life time. It indicates irrespective of change in pause time all the protocols battery drain time not changing more. In this simulation pause time is varied by keeping maximum node speed as a constant. For our convienience we change the axis from 0-1200 to 1197.9- 1198.5 because we take the simulation time of $300 \mathrm{~s}$.

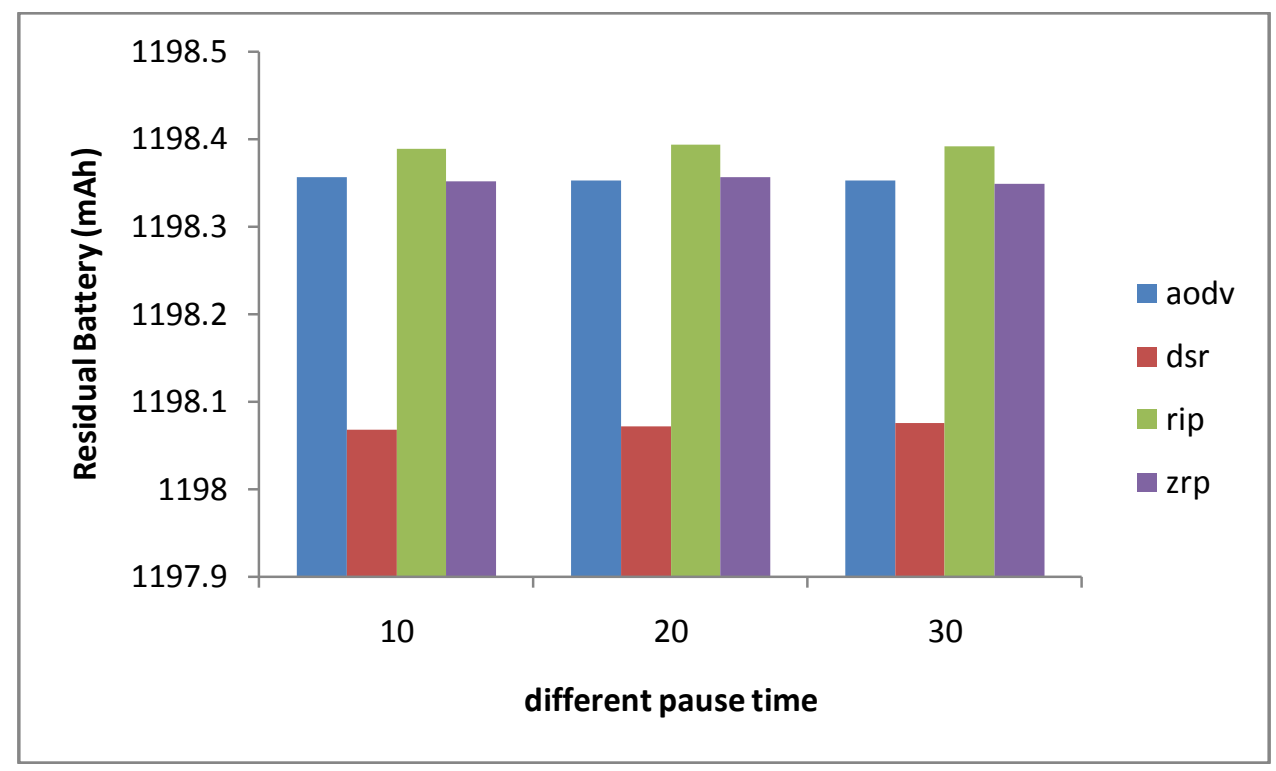

\section{Conclusions}

In this paper we compare four different routing protocol(aodv,dsr,rip \& zrp) on basis of their network lifetime.since the lifetime of the network will depends on the battey capacity of the nodes.As the battery discharge the network is no longer live \& all the connection through this network is going cut off. In this paper we compare the network for different cbr, mobility and pause time. It is observed that Rip lifetime is better than the other three routing protocol. In these network condition it is found that Rip is always superior in term of node mobility \& increase traffic. With this network lifetime analysis we agreed with other study that Rip is the standard routing protocol for energy constraint short MANETs.

\section{References}

[1] [Routing Information Protocol (RIP),june 1988, The Internet Society http://tools.ietf.org/html/rfc1058

[2] [Z. Haas and M. Pearlman, "Determining the Optimal Configuration for the Zone Routing rotocol", IEEE JSAC, Special Issue on Ad-Hoc Networks, August 1999.]

[3] [The Dynamic Source Routing Protocol for Mobile Ad Hoc Networks (DSR). Routing in Mobile Ad Hoc Networks. Internet EngineeringTask Force, Mar. 2001. http://www.ietf.org/internetdrafts/ draft-ietf]

[4]. [Ankit Bhardwaj, Divya and Sanjeev Sofat, "An Efficient Energy Conserving Scheme for IEEE 802.11 ADHOC Networks", IEEE 1-4244-1005-3/07,2007.]

[5] Gomez, J. \& X Campbell, J. (2007). Variable-range transmission power control in wireless ad hocnetworks. IEEE Transaction on mobile computing, vol. 6(1), pp. 87-99.

[6] Wu, S.L \& Tseng, Y.C. (2007). Wireless ad hoc networking, Personal-Area, Local-Area, and thesensory Area Networks, Auerbach publication, ISBN 10: 10: 0-8493-9254-3.]

[7] A.Tsertou and D.I. Laurenson. (2008). Revisiting the Hidden Terminal Problem in a CSMA/CA Wireless Network. IEEE Transactions on Mobile Computing, vol. 7(7), pp.817-831, October 2008.

[8] [A.K.Vatsa, Prince Chauhan, Meenakshi Chauhan and Jyothi Sharma, "Routing Mechanism for MANET in Disaster Area", International Journal of Networking and Mobile Technologies, Vol 2 / ISSUE 2/ MAY 2011.]

[9]. [Mobile Ad Hoc Networking Working Group - AODV, http://www.ietf.org/rfc/rfc3561.txt].

[10] Niranjan kumar Ray,Harsh Bardhan Sharma \& Ashok kumar Turuk, "Network Lifetime Analysis of AODV, DSR andZRP at Different Network Parameters" International Journal of Mobile Network Communications \& Telematics (IJMNCT) Vol.2, No.3, June 2012 